\title{
Primary Bile Acid Malabsorption Caused by Mutations in the lleal Sodium-dependent Bile Acid Transporter Gene (SLC10A2)
}

\author{
Peter Oelkers, ${ }^{*}$ Lyndon C. Kirby, ${ }^{*}$ James E. Heubi, ${ }^{\ddagger}$ and Paul A. Dawson* \\ *Department of Internal Medicine, Division of Gastroenterology, Bowman Gray School of Medicine of Wake Forest University, \\ Winston-Salem, North Carolina 27157; and ${ }^{\ddagger}$ Department of Pediatrics, Children’s Hospital Medical Center, University of Cincinnati, \\ Cincinnati, Ohio 45229
}

\begin{abstract}
Primary bile acid malabsorption (PBAM) is an idiopathic intestinal disorder associated with congenital diarrhea, steatorrhea, interruption of the enterohepatic circulation of bile acids, and reduced plasma cholesterol levels. The molecular basis of PBAM is unknown, and several conflicting mechanisms have been postulated. In this study, we cloned the human ileal $\mathrm{Na}^{+} /$bile acid cotransporter gene (SLC10A2) and employed single-stranded conformation polymorphism analysis to screen for PBAM-associated mutations. Four polymorphisms were identified and sequenced in a family with congenital PBAM. One allele encoded an A171S missense mutation and a mutated donor splice site for exon 3 . The other allele encoded two missense mutations at conserved amino acid positions, L243P and T262M. In transfected COS cells, the L243P, T262M, and double mutant (L243P/ T262M) did not affect transporter protein expression or trafficking to the plasma membrane; however, transport of taurocholate and other bile acids was abolished. In contrast, the A171S mutation had no effect on taurocholate uptake. The dysfunctional mutations were not detected in 104 unaffected control subjects, whereas the A171S was present in $28 \%$ of that population. These findings establish that SLC10A2 mutations can cause PBAM and underscore the ileal $\mathrm{Na}^{+}$/bile acid cotransporter's role in intestinal reclamation of bile acids. (J. Clin. Invest. 1997. 99:1880-1887.) Key words: bile salts $\bullet$ cholesterol $\bullet$ bile acid malabsorption $\bullet$ mutation analysis $\bullet$ genetic disease
\end{abstract}

Portions of this work were presented at the 96th Annual Meeting of the American Gastroenterology Association in San Francisco, CA on 19-22 May 1996. Portions of this work have appeared in abstract form (1996. Gastroenterology. 110:826a).

Address correspondence to Paul A. Dawson, Department of Internal Medicine, Division of Gastroenterology, Bowman Gray School of Medicine, Medical Center Blvd., Winston-Salem, NC 27157. Phone: 910-716-4633; FAX: 910-716-6376. P. Oelkers' present address is Institute of Human Nutrition, Columbia University, New York 10032; L.C. Kirby's present address is Department of Physiology, East Carolina University School of Medicine, Greenville, NC 27834.

Received for publication 31 October 1996 and accepted in revised form 29 January 1997.

J. Clin. Invest.

(C) The American Society for Clinical Investigation, Inc. 0021-9738/97/04/1880/08 \$2.00

Volume 99, Number 8, April 1997, 1880-1887

\section{Introduction}

Bile acids are synthesized from cholesterol in the liver and secreted into the small intestine, where they facilitate absorption of fat, fat-soluble vitamins, and cholesterol (1). The bile acids are reabsorbed from the intestine, returned to the liver via the portal venous circulation, and resecreted into bile (2). This enterohepatic circulation of bile acids is an extremely efficient process, $<10 \%$ of the intestinal bile acids escape reabsorption and are eliminated in the feces. The first step in the active uptake of bile acids from the intestine is mediated by a $\mathrm{Na}^{+}$gradient-driven transporter located on the apical membrane of the ileal enterocyte (1). This $\mathrm{Na}^{+} /$cotransporter was identified by expression cloning (3) and subsequently, the human ileal $\mathrm{Na}^{+} /$bile acid cotransporter cDNA was isolated (4) and its gene (SLC10A2) was localized to chromosome 13q33 (5). In the course of cloning and characterizing the human ileal $\mathrm{Na}^{+} /$ bile acid cotransporter, a dysfunctional missense mutation was also identified (4). To determine if similar mutations are associated with alterations in bile acid metabolism, we have cloned the human ileal $\mathrm{Na}^{+}$/bile acid cotransporter gene and used single-stranded conformation polymorphism (SSCP) ${ }^{1}$ analysis to screen for inherited mutations in a family with primary bile acid malabsorption (PBAM).

PBAM is an idiopathic intestinal disorder associated with chronic diarrhea beginning in early infancy, steatorrhea, interruption of the enterohepatic circulation of bile acids, and reduced plasma cholesterol levels (6-8). While the molecular basis of PBAM is unknown, a specific defect in bile acid transport by the distal ileum has been postulated (7-10). In support of this hypothesis, impaired ileal uptake of bile acids has been documented in several patients $(7,11)$. However, this was not a universal finding (12) and a specific defect in the ileal bile acid transport system has not been demonstrated. This has led others to suggest that PBAM is not a specific transport defect and is a consequence of an enlarged bile acid pool size or increased intestinal motility $(12,13)$. Here, we report that PBAM can be caused by inherited mutations in the ileal $\mathrm{Na}^{+} /$ bile acid cotransporter gene (SLC10A2).

\section{Methods}

Materials and general methods. Genomic DNA was isolated using an SDS-proteinase K procedure (14) or a TurboGen kit (Invitrogen Corp., San Diego, CA). Genomic DNA from Centre d'Etude du Polymorphisme Humain $(\mathrm{CEPH})$ subjects was kindly provided by Dr. Don

1. Abbreviations used in this paper: hISBT, human ileal sodium/bile acid cotransporter; PBAM, primary bile acid malabsorption; SSCP, single-stranded conformation polymorphism. 
Bowden, Bowman Gray School of Medicine. Double stranded DNA templates were sequenced by the dideoxy chain termination method using Sequenase 2.0 (United States Biochemicals, Cleveland, $\mathrm{OH}$ ) and $\left[\alpha{ }^{35} \mathrm{~S}\right] \mathrm{dATP}(600 \mathrm{Ci} / \mathrm{mmol})$ (Amersham Corp., Arlington Heights, IL). $\left[{ }^{3} \mathrm{H}\right]$ Taurocholate $(2.0-2.6 \mathrm{Ci} / \mathrm{mmol}),\left[2,4-{ }^{3} \mathrm{H}\right]$ cholate $(27.5 \mathrm{Ci} /$ $\mathrm{mmol})$, [carboxyl $-{ }^{14} \mathrm{C}$ ]-chenodeoxycholic acid $(48.6 \mathrm{mCi} / \mathrm{mmol})$, and $\left[1-{ }^{14} \mathrm{C}\right]$-glycine ethyl ester hydrochloride $(43.3 \mathrm{mCi} / \mathrm{mmol})$ were purchased from NEN Research Products (Wilmington, DE). Chenodeoxycholic acid and ursodeoxycholic acid were purchased from Calbiochem Corp. (La Jolla, CA). Other unlabeled bile acids were purchased from Sigma Chemical Co. (St. Louis, MO). Triethylamine, $\mathrm{N}$-ethoxycarbonyl-2-ethoxy-1,2-dihydroquinoline, and glycine ethyl ester-HCl were purchased from Aldrich Chemical Co. (Milwaukee, WI). The ${ }^{14} \mathrm{C}$-labeled glycine conjugates of chenodeoxycholic acid, deoxycholic acid, and ursodeoxycholic acid were synthesized as described (15).

Subjects. This study was reviewed and approved by the Clinical Practices Committee of the Bowman Gray School of Medicine and the Institutional Review Board of the Children's Hospital Medical Center. Informed consent was obtained from all study subjects. The PBAM proband has been described previously (7). The control subjects were selected from 160 healthy students (105 males and 55 females, aged 22 to 39) from the Bowman Gray School of Medicine. Blood leukocytes were isolated for genomic DNA isolation. Plasma total cholesterol and triglyceride levels were determined using a Technicon RA-1000 analyzer (Technicon, Tarrytown, NY). HDL cholesterol was measured by heparin-manganese precipitation and the LDL cholesterol level was calculated from total cholesterol, triglyceride, and HDL values using the Friedewald formula (16).

SLC10A2 gene cloning. Three human genomic clones containing ileal $\mathrm{Na}^{+} /$bile acid cotransporter sequences in bacteriophage P1 vectors were obtained from Genome Systems (St. Louis, MO). Human Foreskin Fibroblast P1 Library \#1 (clone addresses 2376, 2377, 2378; DuPont-Merck Pharmaceutical Co., Wilmington, DE) was screened by PCR using primers flanking exon 4, TTTGACTCATGATTGCTGGGTTCAC and CCCAATGTGATTTACTAAATGCCAT. Exoncontaining fragments were identified by hybridization, subcloned into pBluescriptII KS (Stratagene Inc., La Jolla, CA), and sequenced. The intron sizes were determined by long PCR analysis (17) and Southern blotting.

Isolation of a linked CA repeat and genotype analysis. A CA dinucleotide repeat sequence was isolated from $\mathrm{P} 1$ clone 2376 by hybridization with a poly(dA-dC)-poly(dG-dT) probe (Pharmacia LKB Biotechnology Inc., Piscataway, NJ). The $0.25-\mathrm{kb}$ fragment was sequenced and found to encode 17 consecutive CA repeats, $(\mathrm{CA})_{17}$. Primers, CAF (5'-AAGTTATTTTTAGTTCTAATGTTC- $\left.3^{\prime}\right)$ and CAR (5'-GAAACATATATTCATCAGATCAC-3'), were designed and used for simple sequence length polymorphism analysis of genomic DNA. PCR was performed using $50 \mathrm{mM} \mathrm{KCl}, 1.5 \mathrm{mM} \mathrm{MgCl}_{2}$, $20 \mathrm{mM}$ Tris- $\mathrm{HCl}, \mathrm{pH} 8.4,200 \mu \mathrm{M}$ dNTPs, $0.23 \mu \mathrm{M}$ CAF and CAR, $65 \mathrm{nM}$ of CAR labeled at the $5^{\prime}$ end with ${ }^{32} \mathrm{P}$, and $100 \mathrm{ng}$ of human genomic DNA. After an initial denaturation step at $96^{\circ} \mathrm{C}$ for $5 \mathrm{~min}$, PCR amplification was performed for 25 cycles of $94^{\circ} \mathrm{C}$ for $1 \mathrm{~min}$, $55^{\circ} \mathrm{C}$ for $1 \mathrm{~min}$, and $72^{\circ} \mathrm{C}$ for $1 \mathrm{~min}$. Analysis of 78 unrelated Caucasian individuals (CEPH parents) revealed that the marker was polymorphic with at least nine different alleles. The number of repeats and frequencies were as follows: $10,0.09 ; 12,0.006 ; 13,0.17 ; 14,0.36$; $15,0.02 ; 16,0.13 ; 17,0.18 ; 18,0.006$; and $20,0.04$. The heterozygosity was $78 \%$ with a calculated polymorphism information content of 0.75 .

Mutation analysis and sequencing. SSCP analysis was performed as described previously (4). The reactions were resolved on gels containing 6, 8, and $10 \%$ acrylamide (acrylamide: $N, N^{\prime}$-methylenebisacrylamide ratio 50:1) and $8 \%$ acrylamide (acrylamide: $N, N^{\prime}$-methylenebisacrylamide ratio 29:1) to increase the assay sensitivity (18). To identify the nucleotide sequence changes responsible for the SSCP band shifts, the unlabeled PCR products were resolved on a $1.5 \%$ agarose gel, isolated for subcloning, and individual clones (at least eight clones per mutation) were sequenced.
To verify and screen for the exon 3 splice junction mutation, allele-specific PCR (19) was performed using human ileal sodium/bile acid cotransporter (hISBT) 48 (CTGATCACATTGCCCTTCTGTCAT) and an antisense oligonucleotide, hISBT 47M (5'-TGGTGTGAACTGGGATACTTAAAG-3'), whose 3' end was complementary to the trinucleotide substitution at the exon 3 splice junction in the proband (JB). PCR amplification and MboII restriction endonuclease digestion (Promega Corp., Madison, WI) were used to assay for the RFLP in exon 4 caused by the L243P mutation. PCR amplification was performed with $100 \mathrm{ng}$ of genomic DNA, exon 4 flanking oligonucleotides, and $5 \mu \mathrm{Ci}\left[{ }^{32} \mathrm{P}\right] \mathrm{dCTP}$. Aliquots of the ${ }^{32} \mathrm{P}$-labeled PCR product were digested with $\mathrm{MboII}$ and analyzed on a $6 \%$ polyacrylamide gel containing $1 \times$ TBE buffer.

Site-directed mutagenesis and COS cell expression. A PCR-based strategy employing $P f u$ polymerase (Stratagene Inc.) was used for site-directed mutagenesis (20). The constructs were subcloned into pCMV5, and the complete insert was sequenced. For bile acid uptake assays, COS cells were transfected and assayed for radiolabeled bile acid uptake and ileal $\mathrm{Na}^{+}$/bile acid cotransporter protein expression as described (4). To assess the cell surface expression of wild-type and mutant ileal $\mathrm{Na}^{+} /$bile acid cotransporter proteins, cell surface biotinylation was performed using transfected COS cells (4). As a control for specificity and cell membrane integrity, biotinylation of the endogenously expressed and abundant cytosolic protein, Hsp 70, was also examined. Extracts from the biotinylated cells were subjected to immunoprecipitation as described (4), except $1.0 \mu \mathrm{g}$ of a mouse anti-human Hsp 72/73 antibody (21) (Boehringer Mannheim Biochemicals, Indianapolis, IN) was used and $0.1 \%$ (wt/vol) SDS was included in the immunoprecipitation and wash buffers. After SDS-PAGE and transfer to nitrocellulose, the blots were probed using $0.2 \mu \mathrm{g} / \mathrm{ml}$ of the mouse anti-Hsp 72/73 antibody and a horseradish peroxidase-conjugated sheep anti-mouse antibody (1:2,500 dilution; Amersham Corp.), or probed directly with horseradish peroxidase-conjugated streptavidin. The blots were visualized using an enhanced chemiluminescent detection system (ECL; Amersham Corp.).

\section{Results}

Primary bile acid malabsorption. The phenotype of the proband has been described previously $(6,7,22-24)$. Diarrhea and steatorrhea began on day 2 after the patient's birth in 1973. At age $13 \mathrm{mo}$, he presented to Children's Hospital with anasarca, protuberant abdomen, and a rash compatible with zinc deficiency. His height and weight were below the 5 th percentile. Further analysis revealed an increased stool output $(282 \mathrm{~g} / \mathrm{d})$, increased steatorrhea (coefficient of fat absorption, $47.8 \%$ ), markedly increased fecal bile acid excretion, an increased fractional turnover rate for bile acids, and a decreased bile acid pool size (7). The steatorrhea (coefficient of fat absorption, $90 \%$ ) and stool weight (142 g/d) decreased after placement on a medium chain triglyceride formula. $\mathrm{He}$ has continued to have bulky stools but has grown normally; at age 16, his weight was $60.9 \mathrm{~kg}$ (25th percentile) and his height was $168.8 \mathrm{~cm}(10$ 15 th percentile). The proband's plasma LDL cholesterol levels have consistently remained below the 10th percentile. In contrast, the patient's brother had a normal plasma cholesterol profile and no history of diarrhea or symptomatic gastrointestinal disease. The proband's parents were deceased at the time of this analysis, but had no history of gastrointestinal disease. The proband's son is asymptomatic and the kinetics of bile acid turnover have not been analyzed in this subject.

Structure and organization of the ileal $\mathrm{Na}^{+} /$bile acid transporter gene. To facilitate analysis of the human ileal $\mathrm{Na}^{+} / \mathrm{bile}$ acid cotransporter as a candidate gene for PBAM, the $S L C 10 A 2$ gene was cloned and characterized. The $S L C 10 A 2$ gene is or- 
A

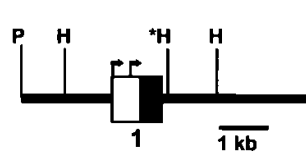

\section{E}
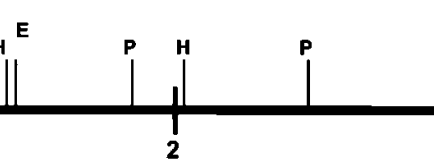

B

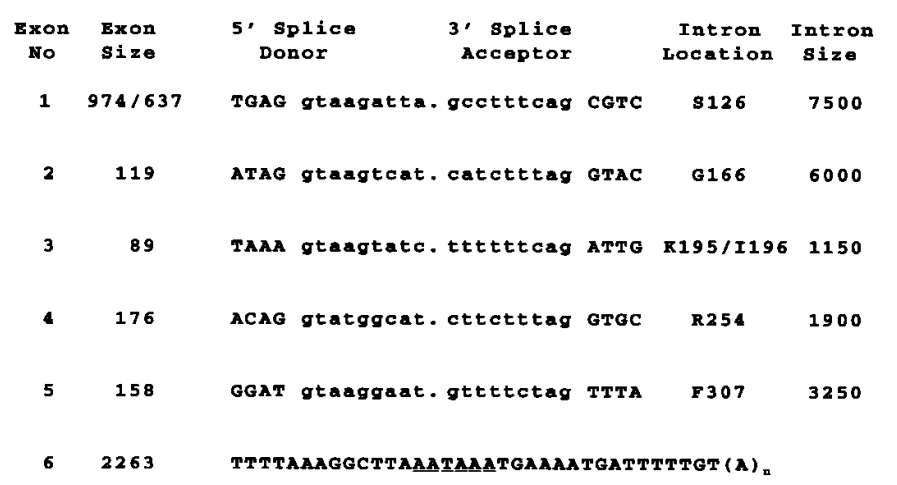

Figure 1. Structure of the human $S L C 10 A 2$ gene. (A) Exons are represented by the boxes and introns by the thick lines. The coding sequence in each exon is indicated by the shaded boxes, the $5^{\prime}$ and $3^{\prime}$ untranslated regions are denoted by the open boxes. The two transcription initiation sites are indicated by the small arrows. Restriction endonuclease sites used to map the gene and $\mathrm{P} 1$ clones are indicated by the vertical lines. H, HindIII; E, EcoRI; $\mathrm{P}$, PstI. Asterisks indicate polymorphic restriction endonuclease sites. $(B)$ The nucleotide sequences of the exon/intron junctions are indicated. Exon sequences are shown in uppercase letters, intron sequences are in lowercase. The size of the introns was estimated by long PCR

amplification and Southern blotting. Two alternate transcription start sites are used yielding two different sizes for exon one. For exon 6, the polyadenylation consensus sequence is underlined and the poly(A) tract is indicated. The nucleotides sequences have been submitted to the Genbank/EMBL/DDBJ data bank with accession numbers U67669-U67674.

ganized in 6 exons spanning $\sim 24 \mathrm{~kb}$ of DNA sequence (Fig. 1 $A$ ). The exon/intron organization of the human $S L C 10 A 2$ gene is shown in Fig. $1 \mathrm{~B}$. The size of each exon and intron, the sequence at the exon/intron junctions, and the amino acid interrupted at each junction are indicated. The $S L C 10 A 2$ gene uses two transcription initiation sites located $\sim 337$ nucleotides apart (Craddock, A.L., M.W. Love, R.W. Daniel, L.C. Kirby, H.C. Walters, M.H. Wong, and P.A. Dawson, manuscript sub- mitted for publication). The first exon encodes the $5^{\prime}$ untranslated region and amino acids 1 to 126 . Exons 2-6 encompass the remaining coding sequence (amino acids 126 to 348). Exon 6 also encodes a long $3^{\prime}$ untranslated region of 2,134 nucleotides.

Analysis of a patient with primary bile acid malabsorption. As an initial screen to determine if mutations in SLC10A2 are associated with PBAM, we identified a polymorphic dinucle-
III

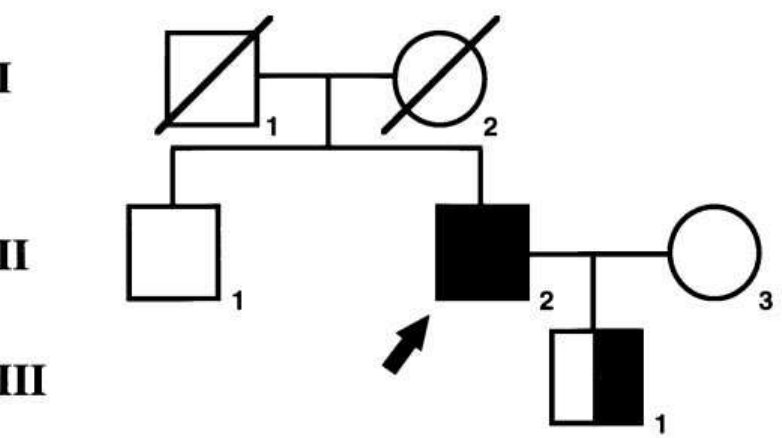

Exon 3

II.1 II.2 III.1 II.3

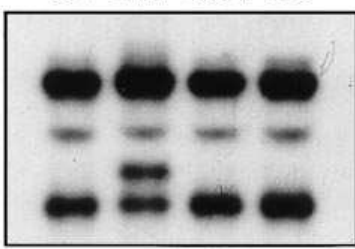

SNA

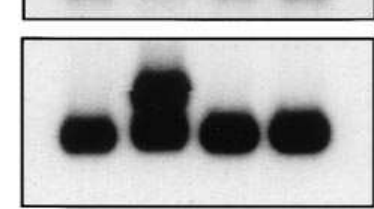

Exon 4

II.1 II.2 III. 1 II.3
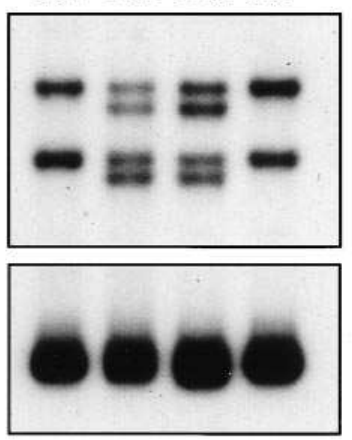

Exon 5

II. 1 II.2 III. 1 II.3
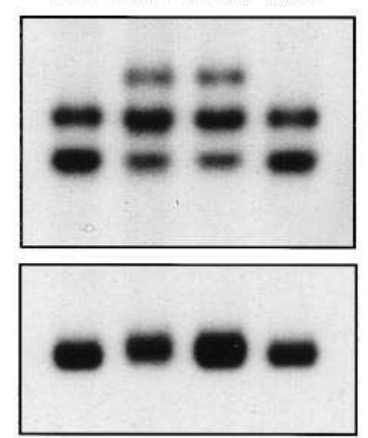

Figure 2. Identification of mutations in the SLC10A2 gene in a family with PBAM. Individuals with SLC10A2 mutations are indicated by the shaded symbols, the proband (II.2) is indicated by the arrow. Genomic DNA from individual family members was prepared from lymphocytes and used for SSCP analysis. The upper boxes show the migration of the single stranded DNA conformers (ss DNA); the lower boxes show the migration of the reannealed double stranded DNA (ds DNA). 
A
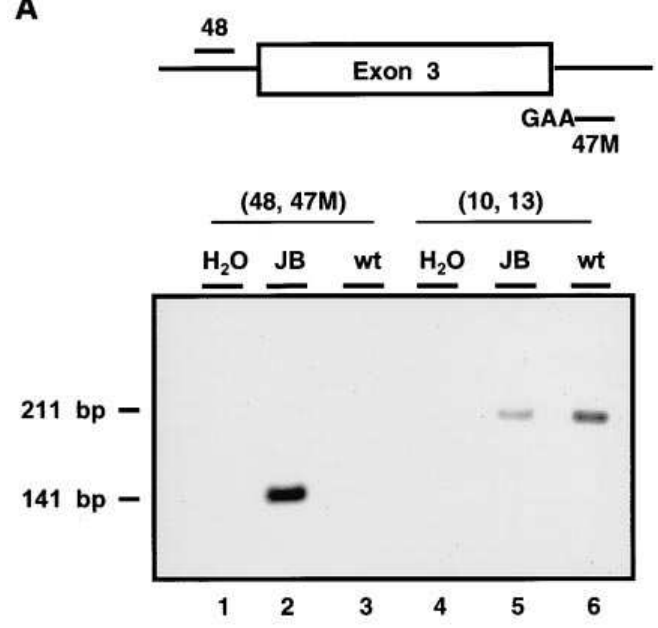

B
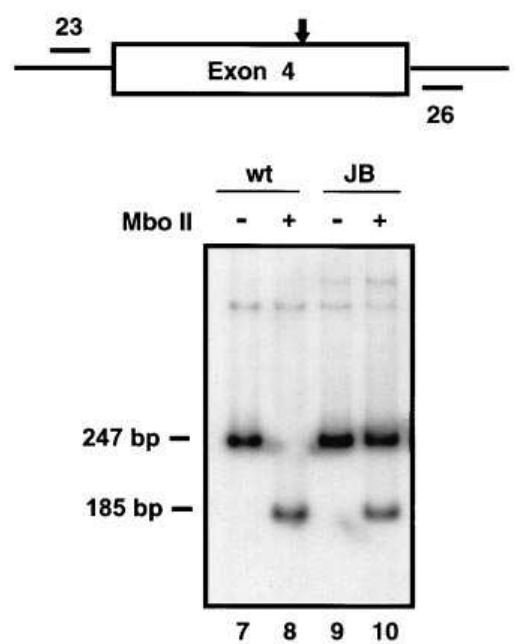

Figure 3. Verification of mutations in $S L C 10 A 2$ gene. (A) Allele-specific PCR was used to verify the trinucleotide substitution at the exon 3 splice junction in JB. The $3^{\prime}$ terminus of oligonucleotide hISBT 47M is complementary to the CTT substitution. PCR was performed with exon 3-specific primers hISBT 48 and 47M (lanes 1-3), or exon 5-specific primers hISBT 10 and 13 (lanes 4-6) with $100 \mathrm{ng}$ of the indicated genomic DNA samples. The products were resolved on a $2 \%$ agarose gel and stained with ethidium bromide. $(B)$ MboII restriction enzyme analysis of $S L C 10 A 2$. Exon 4 was PCR amplified from control (lanes 7 and 8 ) or JB genomic DNA (lanes 9 and $10)$ in the presence of $\left[{ }^{32} \mathrm{P}\right] \mathrm{dCTP}$.

The PCR products were then incubated in the absence (lanes 7 and 9) or presence (lanes 8 and 10) of MboII and resolved on a $6 \%$ acrylamide gel. The dried gel was exposed to $\mathrm{x}$-ray film for $3 \mathrm{~h}$ at $-70^{\circ} \mathrm{C}$ with an intensifying screen.

otide repeat linked to the $S L C 10 A 2$ gene. Simple sequence length polymorphism analysis of genomic DNA from a PBAM family revealed that the proband, JB, carried at least one different $S L C 10 A 2$ allele from his unaffected brother (data not shown). Thus, $S L C 10 A 2$ could not be excluded as a candidate gene for this putative autosomal recessive disorder and SSCP analysis was then employed. Polymorphisms were identified in exons 3-5. SSCP analysis of the proband's family revealed that the polymorphisms in exons 4 and 5 lie on one allele and the exon 3 polymorphism(s) on the other (Fig. 2).

To determine the sequence changes responsible for the novel bands observed by SSCP, the exons harboring the polymorphisms were PCR amplified and subcloned, and multiple clones were sequenced. JB was heterozygous for each of the polymorphisms as evidenced by finding both wild-type and polymorphic sequences among the PCR products (data not shown). These studies confirmed that exon 3 harbors two polymorphisms. The heteroduplex detected by SSCP analysis was due to an unusual 3-bp substitution at the donor splice site of exon 3 that changed the sequence from AAg to CTt. To verify that this trinucleotide substitution was not a cloning or sequencing artifact, allele-specific PCR was performed. As shown in Fig. $3 A$, PCR analysis of genomic DNA from JB, but not a control subject, yielded a product with the allele-specific oligonucleotide $47 \mathrm{M}$. The presence of genomic DNA in the control sample was verified by PCR using a pair of oligonucleotides specific for exon 5. A second polymorphism was also identified in exon 3 that encoded a $\mathrm{G}$ to $\mathrm{T}$ transversion result-

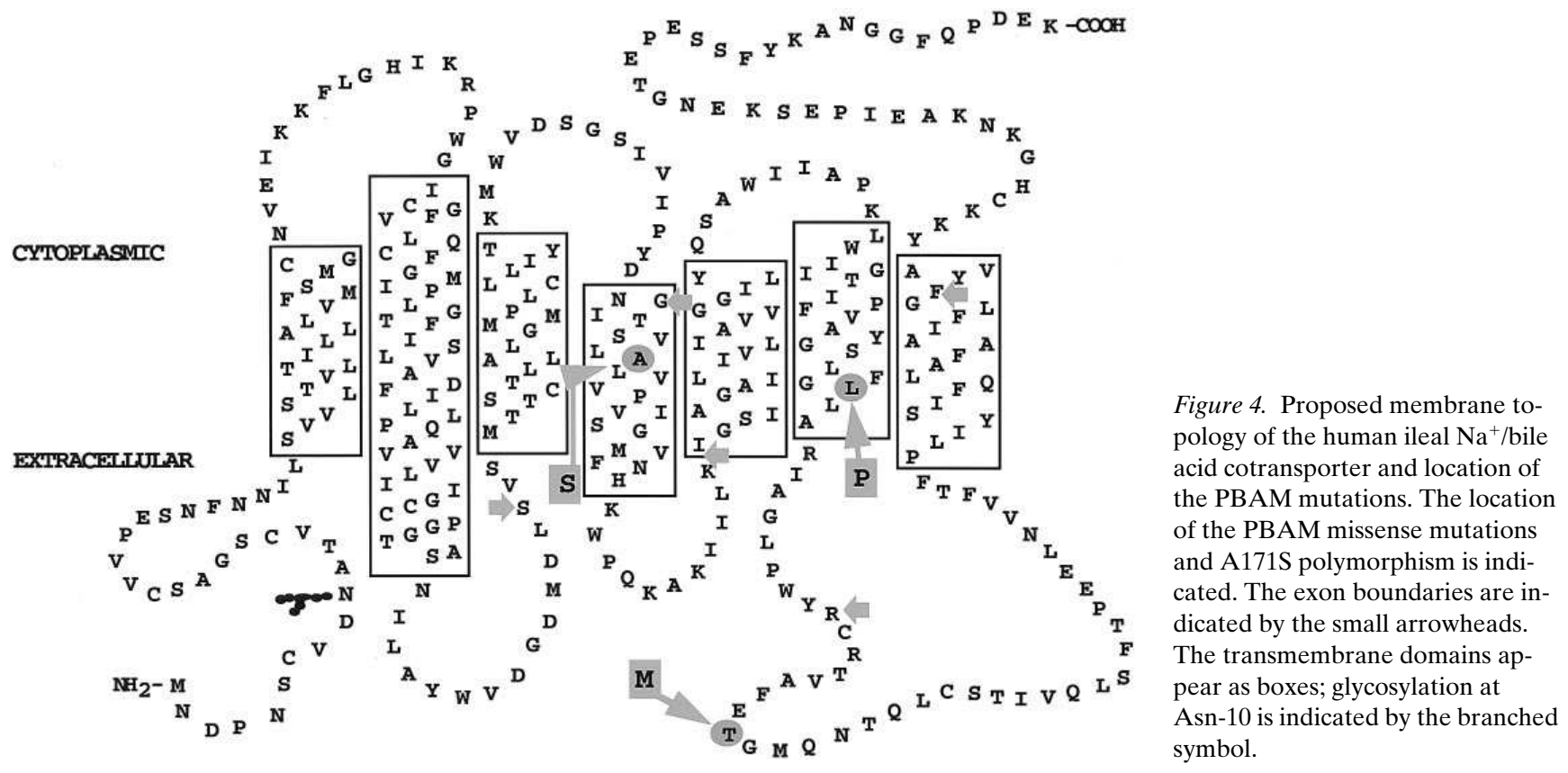


ing in an alanine to serine substitution (A171S) in the transporter's third predicted transmembrane domain (Fig. 4).

The other allele in JB encoded two missense mutations affecting conserved amino acid codons. In exon 4 , a $\mathrm{T}$ to $\mathrm{C}$ transition results in a leucine to proline change at amino acid position 243 (Fig. 4). This mutation also destroyed an MboII restriction endonuclease site. Subsequent MboII enzyme digestion of PCR-amplified genomic DNA from JB confirmed the presence of the mutation (Fig. $3 \mathrm{~B}$ ). In exon 5 , a $\mathrm{C}$ to $\mathrm{T}$ transition was found that causes a threonine to methionine substitution at codon 262 (Fig. 4).

To determine if these polymorphisms were also found in unaffected subjects with normal ileal bile acid absorption, 104 healthy individuals (free of clinical gastrointestinal disease) were screened by SSCP for the presence of the A171S, L243P, T262M, and exon 3 splice junction mutations. LDL cholesterol levels were used as a surrogate marker for ileal $\mathrm{Na}^{+} /$bile acid cotransporter function. As described above, the proband's LDL cholesterol level was consistently below the 10th percentile (age and sex adjusted). Increased loss of bile acids due to ileal dysfunction, ileal bypass, or the use of bile acid sequestrants is a well-established mechanism to lower plasma LDL cholesterol levels due to the diversion of hepatic cholesterol to bile acid synthesis $(25,26)$. Since a defective $S L C 10 A 2$ allele may function like an ileal bypass to lower plasma cholesterol levels, only those control individuals whose plasma LDL cholesterol levels were within $1 \mathrm{SD}$ of the population mean were included in the analysis (27). In the screen for the A171S polymorphism, 75 subjects were alanine 171 homozygotes, 27 subjects were A171S heterozygotes, and 2 subjects were serine 171 homozygotes. Thus, A171S appears to be a common allele that is present in Hardy-Weinberg equilibrium. The two serine 171 homozygotes had no history of gastrointestinal disease or bile acid malabsorption. In contrast with the A171S polymorphism, the exon 3 splice junction mutation and missense mutations in exons 4 and 5 were not found in any of the control individuals.

SLC10A2 mutations account for the lack of ileal bile acid transport activity. The unusual exon 3 splice junction substitution is predicted to cause aberrant splicing of that $S L C 10 A 2$ allele in JB. Similar point mutations at splice donor sites have been found to lead preferentially to exon skipping, and more rarely to cryptic splice site usage (28). To determine if the mis- sense mutations on the other SLC10A2 allele also contribute to the PBAM phenotype, the double mutation L243P/T262M was generated by site-direct mutagenesis, transfected into COS cells, and assayed for bile acid transporter expression and activity. While the double mutant (L243P/T262M) did not affect ileal $\mathrm{Na}^{+} /$bile acid cotransporter protein expression (Fig. 5 $A$, inset), taurocholate transport activity was abolished in the transfected COS cells (Fig. $5 A$ ). Analysis of the individual missense mutations revealed that both the L243P and T262M mutations abolished taurocholate transport activity without affecting ileal $\mathrm{Na}^{+} /$bile acid cotransporter protein expression. In contrast, the common polymorphism A171S did not affect ileal $\mathrm{Na}^{+} /$bile acid cotransporter protein expression or taurocholate uptake in transfected COS cells (data not shown).

These studies show that taurocholate transport is impaired, but do not exclude the possibility that the mutant ileal $\mathrm{Na}^{+}$/ bile acid cotransporter may still affect the transport of other bile acid species. To address this question, expression plasmids for the wild-type and (L243P/T262M) mutant ileal $\mathrm{Na}^{+} /$bile acid cotransporters were transfected into COS cells and assayed for bile acid uptake. As shown in Fig. 6, the mutations also abolished transport of the unconjugated trihydroxy bile acid, cholate, and the conjugated dihydroxy bile acids glycochenodeoxycholate and glycodeoxycholate. Similar results were also observed when the uptake assay was performed using $50 \mu \mathrm{M}$ of these radiolabeled bile acids or when $\left[{ }^{14} \mathrm{C}\right]$ glycoursodeoxycholate was used (data not shown).

To determine if the lack of transport activity for the mutants was due to a block in normal plasma membrane expression, a cell surface biotinylation assay was employed with transfected COS cells. By immunoblotting analysis, similar amounts of wild-type and mutant ileal $\mathrm{Na}^{+} /$bile acid cotransporter proteins were detected in cell extracts from the surface biotinylated COS cells (Fig. 7, lanes 2-5). Analysis of the cell surface expression by immunoprecipitation and streptavidin blotting also detected similar amounts of wild-type and mutant protein (Fig. 7, lanes 7-10). A parallel immunoprecipitation using mouse anti-Hsp 72/73 and streptavidin blotting analysis did not detect biotinylation of the abundant cytosolic protein, Hsp 70, in these same COS cell extracts, whereas this protein was readily detected by immunoblotting (data not shown). Thus, leakage of NHS-LC biotin into the cell could not account for the label-
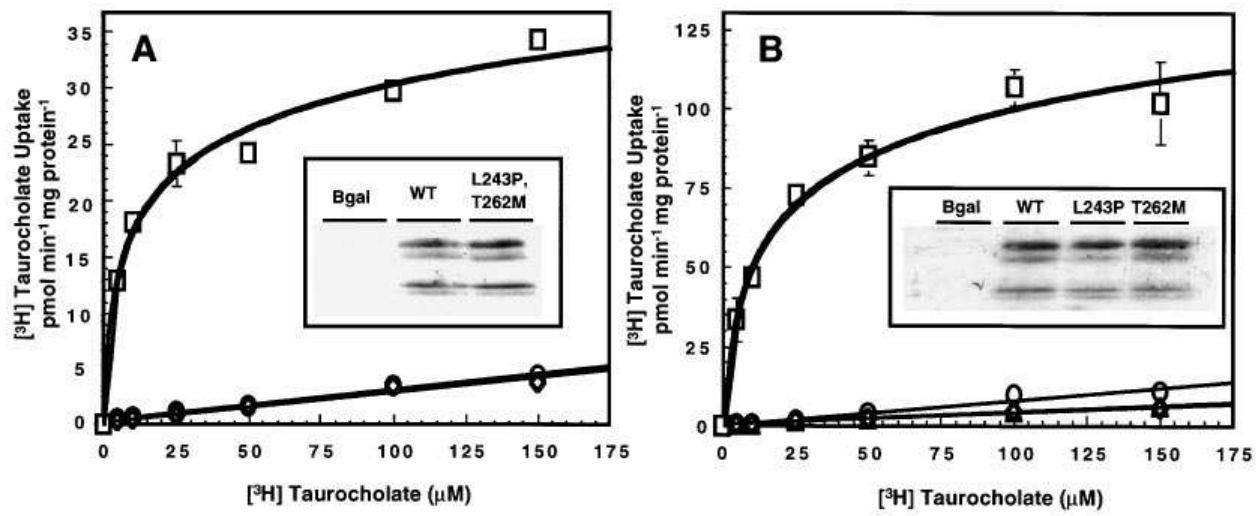

Figure 5. Effect of PBAM-associated ileal $\mathrm{Na}^{+} /$bile acid cotransporter mutations on $\left[{ }^{3} \mathrm{H}\right]$ taurocholate uptake in transfected COS cells. On day 1 , COS cells were transfected with the indicated expression plasmid construct. On day 4 , the cells were incubated in a modified Hanks buffer supplemented with the indicated concentration of $\left[{ }^{3} \mathrm{H}\right]$ taurocholate for $10 \mathrm{~min}$ at $37^{\circ} \mathrm{C}$. Cell monolayers were then washed and processed to determine cellassociated protein and radioactivity. Each value represents the mean $\pm \operatorname{SD}(n=3)$. (Inset) $20 \mu \mathrm{g}$ of cell extract from a parallel dish of transfected COS cells was analyzed by immunoblotting using an antiileal $\mathrm{Na}^{+} /$bile acid cotransporter antibody. (A) $\beta$-Galactosidase $(\Delta)$, wild-type ileal $\mathrm{Na}^{+} /$bile acid cotransporter $(\square)$, and L243P/T262M double mutant $(O)$. $(B) \beta$-Galactosidase $(\Delta)$, wildtype ileal $\mathrm{Na}^{+}$/bile acid cotransporter $(\square)$, L243P mutant $(\diamond)$, and T262M mutant $(\bigcirc)$. 


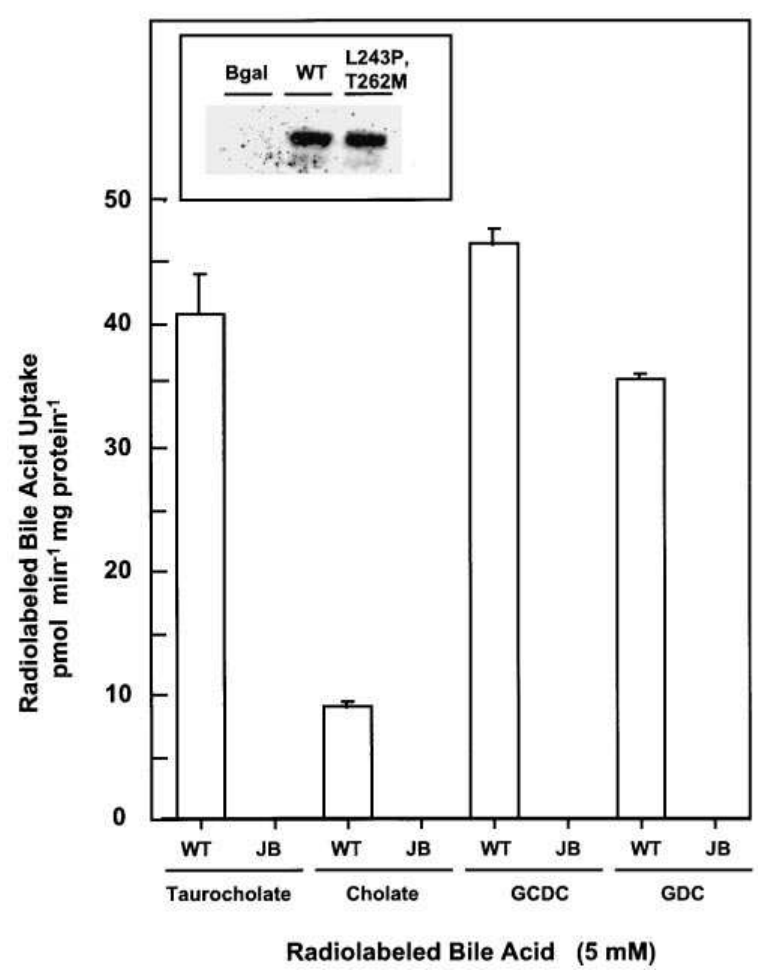

Figure 6. Effect of PBAM-associated ileal $\mathrm{Na}^{+} /$bile acid cotransporter mutations on radiolabeled bile acid uptake in transfected COS cells. COS cells were transfected with plasmids expressing $\beta$-galactosidase, wild-type ileal $\mathrm{Na}^{+} /$bile acid cotransporter $(W T)$, or L243P/ $\mathrm{T} 262 \mathrm{M}$ double mutant $(J B)$. On day 4 , the cells were incubated in a modified Hanks buffer supplemented with $5 \mu \mathrm{M}$ of the indicated radiolabeled bile acid for $10 \mathrm{~min}$ at $37^{\circ} \mathrm{C}$. Cell monolayers were then washed and processed to determine cell-associated protein and radioactivity. The uptake values were corrected for nonspecific uptake by mock (pCMV2- $\beta$-galactosidase) transfected cells and represent the mean $\pm \mathrm{SD}(n=3)$. (Inset) $15 \mu \mathrm{g}$ of cell extract from a parallel dish of transfected COS cells was analyzed by immunoblotting using an antiileal $\mathrm{Na}^{+}$/bile acid cotransporter antibody. GCDC, glycochenodeoxycholate; GDC, glycodeoxycholate.

ing of the wild-type and mutant ileal $\mathrm{Na}^{+} / \mathrm{bile}$ acid cotransporters. These studies indicate that the L243P and T262M mutations do not interfere with ileal $\mathrm{Na}^{+}$/bile acid cotransporter expression on the cell surface, but must interfere in the bile acid binding or transport process.

\section{Discussion}

Primary bile acid malabsorption was first described in a series of adult patients with idiopathic chronic diarrhea that responded to cholestyramine (8). Subsequent follow-up studies documented increased fecal bile acid excretion in the absence of changes in intestinal transit rate, ileal disease, or an infectious etiology $(29,30)$. A more severe form of PBAM was found in infants where, in contrast with the adult PBAM patients, the depletion of the bile acid pool lead to significant steatorrhea and reduced solubilization of dietary lipid. The unabsorbed hydroxy fatty acids act as potent cathartic agents and are primarily responsible for the refractory diarrhea in these patients $(6,7,11,31)$. Numerous radiographic studies and in-

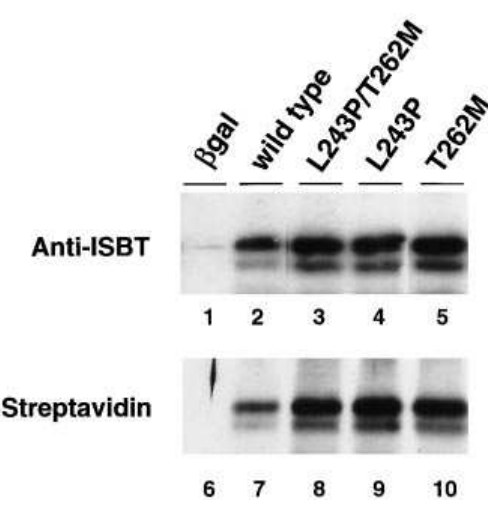

Figure 7. Immunoblotting and streptavidin detection of biotinylated wild-type and mutant human ileal $\mathrm{Na}^{+} /$bile acid cotransporters in transfected COS cells. Detergent lysates were prepared from transfected COS cells after cell surface biotinylation. Extracts from equivalent numbers of cells $\left(10^{4}\right)$ were subjected to SDS-PAGE on a $10 \%$ acrylamide gel and immunoblotting with rabbit antiileal $\mathrm{Na}^{+} /$ bile acid cotransporter antibody $(1.8 \mu \mathrm{g} / \mathrm{ml})$. The primary antibody was detected using horseradish peroxidase-conjugated donkey antirabbit antibody, and visualized by ECL (lanes 1-5). The remaining cell extract was immunoprecipitated with rabbit antiileal $\mathrm{Na}^{+} / \mathrm{bile}$ acid cotransporter antibody. Immunoprecipitates corresponding to $7 \times 10^{5}$ cells were subjected to SDS-PAGE on a $10 \%$ acrylamide gel, transferred to nitrocellulose, and probed using horseradish peroxidase-conjugated streptavidin. The streptavidin conjugate was visualized by ECL (lanes $6-10$ ).

testinal biopsies of these children showed a normal small intestine with no indication of inflammation or ileal disease. Studies performed using ileal mucosal biopsies from these patients also showed a diminished uptake of bile acids $(7,11)$. While these results support the hypothesis that PBAM is caused by a congenital defect of active bile acid absorption in the distal ileum (7-10), this explanation is not universally accepted (12, 13). Other studies employing a taurocholate uptake assay with crude brush border membranes prepared from ileal biopsies did not find impaired bile acid uptake in a number of adult patients with primary bile acid diarrhea (12). From these studies, it was concluded that PBAM is not caused by a defect in active ileal bile acid transport, but is a consequence of motor abnormalities resulting in increased intestinal motility $(12,13,32)$.

We present here the genomic cloning of the human ileal $\mathrm{Na}^{+} /$bile acid cotransporter gene, SLC10A2, and demonstrate that mutations in this gene can cause PBAM. The organization of the ileal $\mathrm{Na}^{+} /$bile acid cotransporter gene is almost identical to the homologous rat liver $\mathrm{Na}^{+}$/bile acid cotransporter (33). The introns fall at or near the homologous amino acid positions for the two genes with the exception that exon 2 of the liver bile acid transporter is further subdivided in the ileal $\mathrm{Na}^{+} /$ bile acid cotransporter by an intron at codon 166 . At the amino acid level, exon 5 (amino acids 254-307) shows the greatest identity $(50 \%)$, with the corresponding liver bile acid transporter exon (exon 4). This region encompasses the third putative extracellular loop and the seventh transmembrane domain. The amino acid boundaries for each exon are shown in Figs. $1 B$ and 4 . The amino acid identity between the human ileal and rat liver $\mathrm{Na}^{+} /$bile acid cotransporter for each of the ileal cotransporter exons was: exon $1,35 \%$; exon $2,46 \%$; exon $3,37 \%$; exon $4,21 \%$; exon $5,50 \%$; and exon $6,13 \%$. The overall amino acid identity between the liver and ileal $\mathrm{Na}^{+} /$bile acid cotransporters is $34 \%$.

Analysis of the coding region of $S L C 10 A 2$ from a patient with PBAM revealed four polymorphisms. The A171S muta- 
tion in exon 3 is a common polymorphism in the Caucasian population and does not appear to affect taurocholate transport. In the proband, exon 3 also harbors an unusual three nucleotide substitution at the splice donor site and is predicted to cause exon skipping (28). The origin of this substitution (5'-AAG-3' to $5^{\prime}$-CTT-3') is unclear, but may be due to an inversion of the dyad symmetry that flanks the mutated sequence (5'-TACTTA AAg taagta- $3^{\prime}$; dyad symmetry is underlined; the exon 3 and intron sequences are shown as uppercase and lowercase, respectively). The other $S L C 10 A 2$ allele in JB harbors two missense mutations. Recent data from in vitro experiments (Hallen, S., D. Bayle, P.A. Dawson, and G. Sachs, manuscript in preparation) support the assignment of the amino terminus, the seven transmembrane domains, and carboxyl terminus shown in Fig. 4; however, a comprehensive analysis of the ileal $\mathrm{Na}^{+}$/bile acid cotransporter's structure has not been performed. The nonconservative L243P substitution in predicted transmembrane domain 6 would dramatically alter the folding and structure of the transporter. In contrast, the T262M change is more conservative and falls in a predicted extracellular loop. However, both amino acid positions are conserved in the ileal $\mathrm{Na}^{+}$/bile acid cotransporter from different species (human, rat, hamster, rabbit, mouse), and T262 is conserved between the liver (SLC10A1) and ileal $\mathrm{Na}^{+} /$bile acid cotransporter genes (34). The high degree of conservation at these positions suggest that the loss of function may be due to either mutation. Characterization of the double and single mutants in transfected COS cells confirmed this prediction. The mutations did not interfere with protein expression or trafficking to the cell surface, but blocked the transport of taurocholate and other bile acids. The finding of two different mutations carried by the same $S L C 10 A 2$ allele was unexpected. However, a recent study of the cystic fibrosis transmembrane conductance regulator gene suggests that double mutant alleles may be more common than originally thought. In that study, a systematic search of the entire coding sequence of the cystic fibrosis transmembrane conductance regulator gene found two different mutations carried by the same allele in 4 of 44 cystic fibrosis patients (35).

Our results establish that loss-of-function mutations in SLC10A2 can cause PBAM. An autosomal recessive inheritance was suggested by earlier studies of PBAM patients (7) and is supported by the lack of clinical symptoms in the proband's son (individual III.1) who inherited an ileal $\mathrm{Na}^{+} /$bile acid cotransporter allele encoding the L243P and T262M missense mutations. This study represents only the second reported defect of a $\mathrm{Na}^{+} /$solute cotransporter associated with a disorder, following the $\mathrm{Na}^{+}$/glucose cotransporter in glucose/ galactose malabsorption $(36,37)$. The finding that dysfunctional mutations in SLC1OA2 cause significant bile acid malabsorption and reduced plasma LDL cholesterol levels in the absence of ileal disease underscores the importance of the ileal $\mathrm{Na}^{+}$/bile acid cotransporter for maintenance of the enterohepatic circulation of bile acids and cholesterol homeostasis.

\section{Acknowledgments}

We thank Dr. Helen Hobbs for her advice and assistance with the patient samples. We also thank Martha Love for her assistance with the normal subject DNA samples and acknowledge the excellent technical assistance of Ann Craddock.

This work was supported by an American Gastroenterology As-
sociation/Janssen Pharmaceutical Research Scholar award (P.A. Dawson) and by U.S. Public Health Service grant M01 RR08084 from the General Clinical Research Center Program, National Center of Research Resources from the National Institutes of Health (NIH) (J.E. Heubi). P.A. Dawson is an Established Investigator of the American Heart Association. P. Oelkers was supported by NIH Cardiovascular Pathology National Service Training Award HL07115.

\section{References}

1. Hofmann, A.F. 1994. Intestinal absorption of bile acids and biliary constituents: the intestinal component of the enterohepatic circulation and the integrated system. In Physiology of the Gastrointestinal Tract. L.R. Johnson, editor. Raven Press, New York. 1845-1865.

2. Hofmann, A.F. 1994. Bile acids. In The Liver: Biology and Pathobiology. I.M. Arias, J.L. Boyer, N. Fausto, W.B. Jakoby, D.A. Schachter, and D.A. Shafritz, editors. Raven Press, New York. 677-717.

3. Wong, M.H., P. Oelkers, A.L. Craddock, and P.A. Dawson. 1994. Expression cloning and characterization of the hamster ileal sodium-dependent bile acid transporter. J. Biol. Chem. 269:1340-1347.

4. Wong, M.H., P. Oelkers, and P.A. Dawson. 1995. Identification of a mutation in the ileal sodium-dependent bile acid transporter gene that abolishes transport activity. J. Biol. Chem. 270:27228-27234.

5. Wong, M.H., P.N. Rao, M.J. Pettenati, and P.A. Dawson. 1996. Localization of the ileal sodium-bile acid cotransporter gene to human chromosome 13q33. Genomics. 33:538-540.

6. Balistreri, W.F., J.E. Heubi, and F.J. Suchy. 1983. Bile acid metabolism: relationship of bile acid malabsorption and diarrhea. J. Pediatr. Gastroenterol. Nutr. 2:105-121.

7. Heubi, J.E., W.F. Balistreri, J.D. Fondacaro, J.C. Partin, and W.K. Schubert. 1982. Primary bile acid malabsorption: defective in vitro ileal active bile acid transport. Gastroenterology. 83:804-811.

8. Thaysen, E.H., and L. Pedersen. 1973. Diarrhea associated with idiopathic bile acid malabsorption: fact or fantasy? Dan. Med. Bull. 20:174-177.

9. Small, D.M., R.H. Dowling, and R.N. Redinger. 1972. The enterohepatic circulation of bile salts. Arch. Intern. Med. 130:552-573

10. Danzinger, R.G., A.F. Hofmann, L.J. Schonfield, and J.L. Thistle. 1972. Dissolution of cholesterol gallstones by chenodeoxycholic acid. N. Engl. J. Med. 286:1-8.

11. Jonas, A., A. Diver-Haber, and S. Avigad. 1986. Well-compensated primary bile acid malabsorption presenting as chronic nonspecific diarrhea. J. Pediatr. Gastroenterol. Nutr. 5:143-146.

12. Van Tilburg, A.J.P., F.W.M. de Rooij, J.W.O. van den Berg, and M. van Blankenstein. 1991. Primary bile acid diarrhea without an ileal carrier defect: quantification of active bile acid transport across the ileal brush border membrane. Gut. 32:500-503.

13. Van Tilburg, A.J.P., F.W.M. de Rooij, J.W.O. van den Berg, and M. van Blankenstein. 1992. Primary bile acid malabsorption: a pathophysiological and clinical entity? Scand. J. Gastroenterol. 27(Suppl. 194):66-70.

14. Strauss, W.M. 1994. Preparation and analysis of DNA. In Current Protocols in Molecular Biology. K. Janssen, editor. John Wiley \& Sons Inc., New York. 2.2.1-2.2.3.

15. Tserng, K.-Y., D.L. Hachey, and P.D. Klein. 1977. An improved procedure for the synthesis of glycine and taurine conjugates of bile acids. J. Lipid Res. 18:404-407.

16. Friedewald, W.T., R.I. Levy, and D.S. Fredrickson. 1972. Estimation of the concentration of low-density lipoprotein cholesterol in plasma without use of the preparative ultracentrifuge. Clin. Chem. 18:499-502.

17. Barnes, W.M. 1994. PCR amplification of up to $35-\mathrm{kb}$ with high fidelity and high yield from $\lambda$ bacteriophage templates. Proc. Natl. Acad. Acad. USA 91:2216-2220.

18. Ravnik-Glavac, M., D. Glavac, and M. Dean. 1994. Sensitivity of singlestrand conformation polymorphism and heteroduplex method for mutation detection in the cystic fibrosis gene. Hum. Mol. Genet. 3:801-807.

19. Little, S. 1995. Amplification-refractory mutation system (ARMS) analysis of point mutations. In Current Protocols in Human Genetics. N.C. Dracopoli, J.L. Haines, B.R. Korf, D.T. Moir, C.C. Morton, C.E. Seidman, J.G. Seidman, and D.R. Smith, editors. John Wiley \& Sons Inc., New York. 9.8.19.8.12.

20. Cormack, B. 1994. Mutagenesis by the polymerase chain reaction. In Current Protocols in Molecular Biology. K. Janssen, editor. John Wiley \& Sons Inc., New York. 8.5.7-8.5.9.

21. Beckmann, R.P., L.A. Mizzen, and W.J. Welch. 1990. Interactions of Hsp 70 with newly synthesized proteins: implications for protein folding and assembly. Science (Wash. DC). 248:850-854.

22. Balistreri, W.F., F.J. Suchy, and J.E. Heubi. 1980. Serum bile acid response to a test meal stimulus: a sensitive test of ileal function. J. Pediatr. 96: 582-594.

23. Heubi, J.E., R.D. Soloway, and W.F. Balistreri. 1982. Biliary lipid com- 
position in healthy and diseased infants, children, and young adults. Gastroenterology. 82:1295-1299.

24. Heubi, J.E., N.C. O'Connell, and K.D.R. Setchell. 1992. Ileal resection/ dysfunction in childhood predisposes to lithogenic bile only after puberty. Gastroenterology. 103:636-640.

25. Buchwald, H., R.L. Varco, J.P. Matts, J.M. Long, L.L. Fitch, G.S. Campbell, M.B. Pearce, A.E. Yellin, W.A. Edminston, R.D. Smink, et al. 1990. Effect of partial ileal bypass surgery on mortality and morbidity from coronary heart disease in patients with hypercholesterolemia. Report of the Program on the Surgical Control of the Hypercholesterolemia (POSCH). N. Engl. J. Med. 323: 946-955

26. Lipid Research Clinics Program. 1984. The lipid research clinics coronary primary prevention trial results. I. Reduction in incidence of coronary heart disease. II. The relationship of reduction in incidence of coronary disease to cholesterol lowering. JAMA (J. Am. Med. Assoc.). 251:351-374.

27. Grundy, S.M., and the expert panel of the National Cholesterol Education Program. 1994. National Cholesterol Education Program second report of the expert panel on detection, evaluation, and treatment of high blood cholesterol in adults. Circulation. 89:1329-1445.

28. Krawczak, M., J. Reiss, and D.N. Cooper. 1992. The mutational spectrum of single base-pair substitutions in mRNA splice junctions of human genes: causes and consequences. Hum. Genet. 90:41-54.

29. Thaysen, E.H., and L. Pedersen. 1976. Idiopathic bile acid catharsis. Gut. 17:965-970.
30. Thaysen, E.H. 1985. Idiopathic bile acid diarrhea reconsidered. Scand. J. Gastroenterol. 20:452-456.

31. Heubi, J.E., W.F. Balistreri, J.C. Partin, W.K. Schubert, and C.A McGraw. 1979. Refractory infantile diarrhea due to primary bile acid malabsorption. J. Pediatr. 94:546-551.

32. Van Tilburg, A.J.P., F.W.M. De Rooij, M. Van Blankenstein, J.W.O. Van Den Berg, and E.P. Bosman-Jacobs. 1990. $\mathrm{Na}^{+}$-dependent bile acid transport in the ileum: the balance between diarrhea and constipation. Gastroenterology. 98:25-32.

33. Karpen, S.J., A.-Q. Sun, B. Kudish, B. Hagenbuch, P.J. Meier, M. Ananthanarayanan, and F.J. Suchy. 1996. Multiple factors regulate the rat liver basolateral sodium-dependent bile acid cotransporter gene promoter. J. Biol. Chem. 271:15211-15221.

34. Dawson, P.A., and P. Oelkers. 1995. Bile Acid Transporters. Curr. Opin. Lipidol. 6:109-114.

35. Savov, A., D. Angelicheva, A. Balassopoulou, A. Jordanova, S.N. Arvanitakis, and L. Kalaydjieva. 1995. Double mutant alleles: are they rare? Hum Mol. Genet. 4:1169-1171.

36. Turk, E., B. Zabel, S. Mundlos, J. Dyer, and E.M. Wright. 1991. Glucose/galactose malabsorption caused by a defect in the $\mathrm{Na}^{+} /$glucose cotransporter. Nature (Lond.). 350:354-356.

37. Martin, M.G., E. Turk, M.P. Lostao, C. Kerner, and E.M. Wright. 1996. Defects in $\mathrm{Na}^{+}$/glucose transporter (SGLT1) trafficking and function cause glucose-galactose malabsorption. Nat. Genet. 12:216-220. 